\title{
Treating Insomnia with High Risk of Depression Using Therapist-Guided Digital Cognitive, Behavioral, and Circadian Rhythm Support Interventions to Prevent Worsening of Depressive Symptoms: A Randomized Controlled Trial
}

\author{
Jeanne Leerssen ${ }^{a, b}$ Oti Lakbila-Kamal ${ }^{a, b} \quad$ Laura M.S. Dekkers $^{a}$ \\ Savannah L.C. Ikelaar ${ }^{a} \quad$ Anne C.W. Albers ${ }^{a}$ Tessa F. Blanken ${ }^{a, b}$ Jaap Lancee ${ }^{c, d}$ \\ Glenn J.M. van der Lande ${ }^{a}$ Teodora Maksimovic ${ }^{a}$ Sophie E. Mastenbroek ${ }^{a}$ \\ Joyce E. Reesen ${ }^{a, b}$ Sjors van de Ven ${ }^{a}$ Tanja van der Zweerde ${ }^{e, f}$ \\ Jessica C. Foster-Dingley ${ }^{a}$ Eus J.W. Van Someren ${ }^{a, b, g}$

\begin{abstract}
aDepartment of Sleep and Cognition, Netherlands Institute for Neuroscience (NIN), Royal Netherlands Academy of Arts and Sciences, Amsterdam, The Netherlands; ${ }^{b}$ Department of Integrative Neurophysiology, Center for Neurogenomics and Cognitive Research (CNCR), Amsterdam Neuroscience, VU University, Amsterdam, The Netherlands; ' ${ }^{D e p a r t m e n t}$

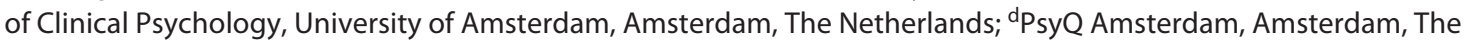
Netherlands; ${ }^{\text {DDepartment }}$ of Psychiatry, Amsterdam Public Health, Amsterdam University Medical Center, Vrije

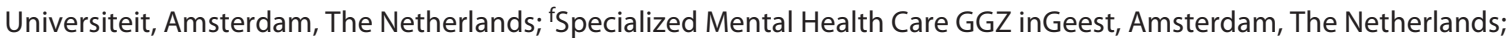
9Department of Psychiatry, Amsterdam UMC, Amsterdam Neuroscience, VU University, Amsterdam, The Netherlands
\end{abstract}

\section{Keywords}

Insomnia treatment · Depressive symptoms · Prevention · Randomized controlled trial

\begin{abstract}
Introduction: The global disease burden of major depressive disorder urgently requires prevention in high-risk individuals, such as recently discovered insomnia subtypes. Previous studies targeting insomnia with fully automated eHealth interventions to prevent depression are inconclusive: dropout was high and likely biased, and depressive symptoms in untreated participants on average improved rather than worsened. Objective: This randomized controlled trial aimed to efficiently prevent the worsening of depressive symptoms
\end{abstract}

karger@karger.com www.karger.com/pps

Karger $\stackrel{\text { ' }}{5}$ GOPEN ACCESS
(C) 2021 The Author(s)

Published by S. Karger AG, Basel

This article is licensed under the Creative Commons Attribution 4.0 International License (CC BY) (http://www.karger.com/Services/ OpenAccessLicense). Usage, derivative works and distribution are permitted provided that proper credit is given to the author and the original publisher. by selecting insomnia subtypes at high risk of depression for internet-based circadian rhythm support (CRS), cognitive behavioral therapy for insomnia (CBT-I), or their combination (CBT-I+CRS), with online therapist guidance to promote adherence. Methods: Participants with an insomnia disorder subtype conveying an increased risk of depression $(n=132)$ were randomized to no treatment (NT), CRS, CBT-I, or CBTI+CRS. The Inventory of Depressive Symptomatology - Self Report (IDS-SR) was self-administered at baseline and at four follow-ups spanning 1 year. Results: Without treatment, depressive symptoms indeed worsened $(d=0.28, p=0.041)$ in high-risk insomnia, but not in a reference group with lowrisk insomnia. Therapist-guided CBT-I and CBT-I+CRS reduced IDS-SR ratings across all follow-up assessments (respectively, $d=-0.80, p=0.001 ; d=-0.95, p<0.001)$. Only
Correspondence to:

Jeanne Leerssen, j.leerssen@vu.nl 
CBT-I+CRS reduced the 1-year incidence of clinically meaningful worsening $(p=0.002)$. Dropout during therapist-guided interventions was very low (8\%) compared to previous automated interventions (57-62\%). Conclusions: The findings tentatively suggest that the efficiency of populationwide preventive strategies could benefit from the possibility to select insomnia subtypes at high risk of developing depression for therapist-guided digital CBT-I+CRS. This treatment may provide effective long-term prevention of worsening of depressive symptoms. Trial registration: the Netherlands Trial Register (NL7359).

(C) 2021 The Author(s)

Published by S. Karger AG, Basel

\section{Introduction}

Since major depressive disorder (MDD) is a highly prevalent disorder with a poor prognosis $[1,2]$, prevention is essential to combat its global burden [3-5]. Preventive strategies would most efficiently be evaluated and implemented in people at risk [6]. A particularly high risk of MDD has recently been found in people with specific types of insomnia disorder (ID) [7] (see online suppl. material for details; for all online suppl. material, see www. karger.com/doi/10.1159/000520282). In brief, insomnia subtypes can be distinguished based on their multivariate profile of stable traits of affect and personality. Interestingly, while subtypes do not differ with respect to sleep complaints, their lifetime risk of MDD varies from $8 \%$ in the least vulnerable ID subtypes up to as much as $54 \%$ in the most vulnerable ID subtypes [7]. This discovery opens up the exciting possibility to select high-risk insomniacs for efficient intervention trials aiming to prevent MDD.

The recommended intervention for insomnia, cognitive behavioral therapy for insomnia (CBT-I) [8], improves both sleep and mood $[9,10]$. Moreover, CBT-I holds promise for scalable implementation because it is also effective when provided digitally [10]. Two studies reported a reduction of depressive symptoms in people with insomnia following fully automated digital CBT-I [11-13]. However, it is difficult to interpret the findings in light of the usefulness of digital CBT-I to prevent worsening of depressive symptoms, because the untreated control group also showed a significant decrease rather than worsening of depressive symptoms. The studies thus demonstrated CBT-I to enhance pending relief of depressive symptoms in people with insomnia rather than prevention of their worsening. A further difficulty with interpreting the findings of these studies on insomnia is the very high dropout rate. Three out of 5 participants did not

Treating Insomnia to Prevent Worsening of Depressive Symptoms complete the 6-week intervention $[11,13]$ and 4 out of 5 were lost to follow-up at 18 months [12]. Among people seeking help for insomnia, those that additionally suffer from more severe symptoms of depression seem more likely to drop out of treatment with CBT targeting insomnia $[14,15]$. In sum, it remains to be investigated whether treatment of insomnia could prevent worsening of depressive symptoms.

The current study aimed to better evaluate the potential of insomnia treatment to prevent worsening of depressive symptoms in the year to follow. Firstly, since prevention would be most efficient in people with the highest risk of developing MDD [6], we selected "high-risk insomniacs," i.e., people with a subtype of ID shown to have a strongly increased probability of lifetime MDD [7], yet without a current diagnosis of MDD. Second, in order to prevent the high dropout rates reported with fully automated digital interventions, while maintaining scalability and cost-benefits relative to face-to-face interventions, we added therapist guidance to the digital interventions. Third, we evaluated whether effects of CBT-I on mood could be strengthened by adding circadian rhythm support (CRS). CRS aims to strengthen the circadian rhythm by means of scheduled bright light exposure, physical activity, and body warming [16-18]. CRS has previously been named chronobiological treatment (CT), but is renamed here to avoid a similar abbreviation as is common for cognitive therapy. Few studies have investigated CRS specifically in ID, with mixed results [19]. One study showed no immediate effect of CRS on sleep, although the addition of CRS to CBT-I helped to maintain improvements induced by CBTI [20]. Several studies showed beneficial effects of CRS on depressive symptoms [21,22].

In case clinically meaningful effects can be demonstrated, offering therapist-guided digital interventions specifically to selected high-risk insomniacs would provide an efficient and scalable contribution to combatting the global disease burden of MDD through prevention.

\section{Materials and Methods}

\section{Study Design Overview}

The study protocol of this randomized controlled trial (RCT) has been previously published [23]. In brief, high-risk insomniacs were randomized to one of four groups: no treatment (NT, passive control), or 6 weeks of digital therapist-guided CRS (active control), CBT-I, or combined CBT-I+CRS. Figure 1 shows a flow diagram of the study. Assessment of outcome measures (see below) took place at baseline (T0) and during four follow-up assessments at week 7 (T1), week 26 (T2), week 39 (T3), and week 52 (T4). This RCT was part of a larger multimodal investigation including, for 


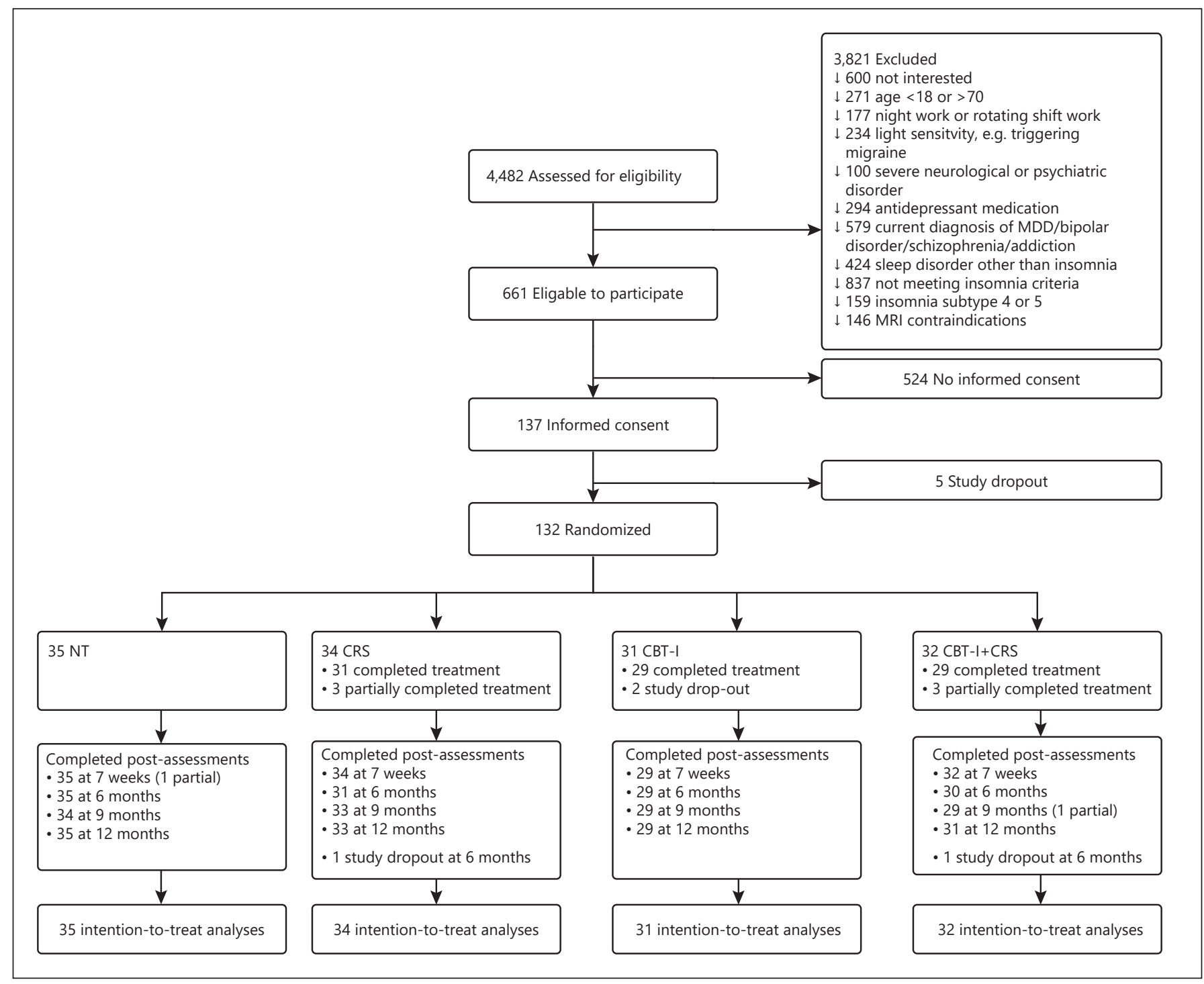

Fig. 1. CONSORT flow diagram. Exclusion criteria were asked sequentially as listed. If an exclusion criterion was met, individuals automatically received a message that they were not eligible to participate, and further exclusion criteria $(\downarrow)$ were not queried. NT, no treatment; CRS, circadian rhythm support; CBT-I, cognitive behavioral therapy for insomnia; CBT-I+CRS, combined cognitive behavioral therapy for insomnia with circadian rhythm support.

example, sleep EEG, MRI, and ambulatory monitoring [23]. Online supplementary Table S2 provides a checklist for methodological recommendations for clinical trials according to Guidi et al. [24].

\section{Participants}

The inclusion criteria were: (1) meeting the criteria of ID according to the 5th edition of the Diagnostic and Statistical Manual of Mental Disorders (DMS-5) [25], and the International Classification of Sleep Disorders, 3rd edition (ICSD-3) [26]; (2) an Insomnia Severity Index (ISI) score $\geq 10[27,28]$; (3) an insomnia type at high risk of depression (type 1,2, or 3) based on the Insomnia Type
Questionnaire [7]; and (4) age between 18 and 70 years. The first exclusion criterion was: (a) a current diagnosis of a depressive disorder, first self-reported during screening, and subsequently confirmed with the Composite International Diagnostic Interview Short Form (CIDI-SF) assessed at baseline. Other exclusion criteria were: (b) current treatment with antidepressant medication; (c) current CBT-I treatment; (d) a severe sleep disorder other than insomnia (i.e., sleep apnea, restless legs syndrome, periodic limb movement disorder); (e) a current diagnosis of any other severe neurological or psychiatric disorder; and (f) night work or rotating shift-work. See Leerssen et al. [23] for more details. 
To ensure generalizability along the guidelines for practical (or pragmatic) clinical trials [29], the use of sleep medication was allowed and monitored, and no cut-off for depressive symptom severity on the Inventory of Depressive Symptomatology - Self Report (IDS-SR) was applied. Online supplementary Table S3 provides a detailed overview of psychotropic medications used and summarizes results of ancillary analyses investigating possible iatrogenic effects. Common depression questionnaire cut-offs overclassify comorbid MDD in insomnia patients in general [30], and especially so in high-risk insomnia subtypes who score high on traits like negative affect [7]. Absence of MDD at baseline was therefore verified with the CIDI structured interview.

Participants were recruited between November 2018 and September 2019 through the Netherlands Sleep Registry (www.sleepregistry.org), media advertisements, and flyers. Of the 4,482 people that visited the website for an online eligibility screening, 137 participants fulfilled all criteria, but 5 withdrew before randomization, resulting in 132 participants.

While the RCT included only participants with high-risk insomnia, T0-T4 assessments were performed in parallel in 30 people with untreated insomnia of a subtype without increased risk of depression $(67 \%$ female, age $=52.6$ years, $\mathrm{SD}=10.9)$. This parallel group allowed for a validation of the expected increased risk in subtype-selected RCT participants.

The study was approved by the Medical Ethics Committee of the VU University Medical Centre (NL63139.029.17) and preregistered, including primary outcomes, at the Netherlands Trial Register (NL7359). All participants provided written informed consent.

\section{Randomization}

Simple randomization was applied until at least 2 participants were randomized to each of the four conditions. Subsequent participants were assigned using scripted covariate-adaptive randomization [31], balancing groups with respect to age, sex, insomnia subtype, baseline severity of insomnia and depressive symptoms, use of sleep medication, suspected sleep complaints other than insomnia (i.e., mild restless legs syndrome and mild sleep apnea), time of year, and group size. Randomization was automated and performed in $\mathrm{R}$ [32] by an independent researcher not involved in any of the assessments, interventions, or analyses (T.F.B.).

\section{Interventions}

The online supplementary material provides details on the digital interventions. Guided by a trained therapist, participants had 6 weeks to complete five sessions of either CRS (i-Cycle) [20], CBT-I (i-Sleep) [33-35], or their combination. Next to the inactive NT condition, CRS may be considered as an active control condition requiring the same amount of time and attention as CBT-I: in a previous study [20] stand-alone CRS had no effect on sleep. Only in combination with CBT-I did CRS support better maintenance of effects that were initially achieved by CBT-I [20].

\section{Outcome measures}

All outcome measures were self-reported or assessed by blinded interviewers. Respecting the importance of incremental validity, our primary and secondary outcome measures were carefully selected and in line with the CLIPROM criteria [24, 36-38]. The preregistered primary outcome was the change, relative to baseline, in severity of depressive symptoms integrated over four follow-ups spanning 1 year. The severity of depressive symptoms was assessed at baseline (T0) and follow-up assessments in week 7 (T1), week 26 (T2), week 39 (T3), and week 52 (T4) using the IDS-SR [39]. IDS-SR ranges indicate no (0-13), mild (14-31), moderate (22-30), severe (31-38), and very severe (39-84) depression [40]. However, we did not use these cut-offs given the lack of validity for use in insomnia patients [30]. Instead, to evaluate the presence of clinically meaningful worsening or improvement on our primary outcome measure at any of the follow-ups, we calculated the Reliable Change Index (RCI) using the baseline IDS-SR score standard deviation and a test-retest reliability of 0.87 $[41,42]$.

A secondary depression outcome was the 12-month incidence of a diagnosis of MDD according to the Composite International Diagnostic Interview - Short Form (CIDI-SF) [43] assessed T4 by trained blinded interviewers.

Secondary outcomes on sleep included: the ISI [28] assessed at T0, T1, T2, T3, and T4; and subjective sleep assessed with the Consensus Sleep Diary [44] at T0 (9 nights), T1 (7 nights), and T4 (7 nights). Sleep diary-derived sleep variables included: sleep efficiency (SE, $0-100 \%$, which is the ratio of total sleep time relative to time in bed; TIB), total sleep time (TST), sleep onset latency (i.e., minutes to fall asleep), and wake after sleep onset (WASO; i.e., minutes awake after sleep onset including time attempting to sleep after final awakening). TIB indicated the time period between closing the eyes to falling asleep, and final awakening when no further attempt to sleep was made [45]. Negative TST and SE values, which can occur when participants overestimate their WASO beyond limits set by TIB, were set to zero.

Side effects were assessed in two ways. First, participants were instructed to report any mild or serious adverse events, serious adverse reactions, and other problems by email. Second, 12-monthretrospective common complaints were assessed both at baseline for reference and at the 12-month follow-up using a validated questionnaire [46].

\section{Sample Size Calculation}

Group sizes of $n=26$ participants completing all five assessments would be sufficient to detect an effect of at least $d=0.5$, with a power of 0.8 and a significance threshold of $\alpha=0.05$ (two-sided) [47]. Expecting dropout and loss to follow-up, a total of 132 participants were included and contributed to intention-to-treat analysis. Dropout and loss to follow-up were less than expected, resulting in 124 participants that fully completed the interventions, and 120 participants that completed each of the five assessments. See the study protocol for more details [23].

\section{Statistical Analyses}

To evaluate how interventions affected IDS-SR-assessed depressive symptom severity throughout the following year, mixed effects regression analysis with two levels (5 assessments nested within subjects) estimated group differences in the change from baseline (T0) to the integrated four follow-up assessments (T1, $\mathrm{T} 2, \mathrm{~T} 3$, and T4). Regressors included dummy coded variables indicating the intervention group, time ( 0 for T0, 1 for T1-T4), and the interaction effects of interest (CRS*time, CBT-I*time, and CBT-I+CRS*time). Intention-to-treat analysis (including all 132 participants), and per-protocol analysis (including 124 partici- 
Table 1. Demographic and clinical characteristics at baseline

\begin{tabular}{|c|c|c|c|c|c|}
\hline & \multicolumn{4}{|c|}{ Mean (SD) or $n(\%)$} & \multirow[t]{2}{*}{$p$ value $^{c}$} \\
\hline & $\begin{array}{l}\text { NT } \\
(n=35)\end{array}$ & $\begin{array}{l}\text { CRS } \\
(n=34)\end{array}$ & $\begin{array}{l}\text { CBT-I } \\
(n=31)\end{array}$ & $\begin{array}{l}\text { CBT-I + CRS } \\
(n=32)\end{array}$ & \\
\hline Age, years & $47.9(12.2)$ & $49.2(13.5)$ & $47.4(13.3)$ & $47.9(12.8)$ & 0.950 \\
\hline Female & $25(71.4 \%)$ & $25(73.5 \%)$ & $23(74.2 \%)$ & $24(75 \%)$ & 0.993 \\
\hline Education in years & $16.1(3.4)$ & $16.8(2.8)$ & $16.5(2.8)$ & $17.2(3.1)$ & 0.505 \\
\hline \multicolumn{6}{|l|}{ Occupation (multiple categories allowed) } \\
\hline Student & $2(5.7 \%)$ & $2(5.9 \%)$ & $1(3.2 \%)$ & $6(18.8 \%)$ & 0.175 \\
\hline Employed & $21(60 \%)$ & $26(76.5 \%)$ & $24(77.4 \%)$ & $23(71.9 \%)$ & 0.385 \\
\hline Unemployed & $12(34.3 \%)$ & $4(11.8 \%)$ & $2(6.5 \%)$ & $3(9.4 \%)$ & 0.013 \\
\hline Retired & $2(5.7 \%)$ & $6(17.6 \%)$ & $4(12.9 \%)$ & $4(12.5 \%)$ & 0.489 \\
\hline Alcohol consumption per week & & & & & 0.488 \\
\hline 0 units of alcohol & $12(34.3 \%)$ & $8(23.5 \%)$ & $5(16.1 \%)$ & $5(15.6 \%)$ & \\
\hline $1-14$ units of alcohol & $21(60.0 \%)$ & $23(67.6 \%)$ & $25(80.6 \%)$ & $24(75.0 \%)$ & \\
\hline $15-21$ units of alcohol & $2(5.7 \%)$ & $3(8.8 \%)$ & $1(3.2 \%)$ & $3(9.4 \%)$ & \\
\hline Smokers & $2(5.7 \%)$ & $3(8.8 \%)$ & $1(3.2 \%)$ & $1(3.1 \%)$ & 0.805 \\
\hline Psychiatric comorbidities $^{a}$ & $2(5.7 \%)$ & $3(8.8 \%)$ & $5(16.1 \%)$ & $1(3.1 \%)$ & 0.288 \\
\hline Sleep medication users & $7(20.0 \%)$ & $7(20.6 \%)$ & $5(16.1 \%)$ & $6(18.8 \%)$ & 0.980 \\
\hline Mild sleep apnea & $2(5.7 \%)$ & $2(5.9 \%)$ & $1(3.2 \%)$ & $1(3.1 \%)$ & 0.999 \\
\hline Mild restless leg syndrome & $3(8.6 \%)$ & $5(14.7 \%)$ & $5(16.1 \%)$ & $4(12.5 \%)$ & 0.798 \\
\hline Insomnia subtypes & & & & & 0.925 \\
\hline Subtype 1 & $10(28.6 \%)$ & $6(17.6 \%)$ & $8(25.8 \%)$ & $7(21.9 \%)$ & \\
\hline Subtype 2 & $17(48.6 \%)$ & $21(61.8 \%)$ & $18(58.1 \%)$ & $18(56.3 \%)$ & \\
\hline Subtype 3 & $8(22.9 \%)$ & $7(20.6 \%)$ & $5(16.1 \%)$ & $7(21.9 \%)$ & \\
\hline Insomnia duration in years & $10.8(10.7)$ & $9.8(11.8)$ & $8.4(10.1)$ & $9.8(11.4)$ & 0.867 \\
\hline Insomnia severity (ISI) & $16.0(4.2)$ & $15.8(3.5)$ & $16.4(4.1)$ & $15.9(3.5)$ & 0.937 \\
\hline Sleep quality (PSQI) & $10.2(3.4)$ & $10.6(3.2)$ & $10.9(3.3)$ & $10.8(3.4)$ & 0.839 \\
\hline Depressive symptoms severity (IDS-SR) & $19.4(6.2)$ & $19.1(7.9)$ & $19.5(8.2)$ & $18.7(7.5)$ & 0.967 \\
\hline Lifetime MDD/dysthymia diagnosis ${ }^{\mathrm{b}}$ & $20(57.1 \%)$ & $19(55.9 \%)$ & $18(58.1 .0 \%)$ & $17(53.1 \%)$ & 0.981 \\
\hline Anxiety (BAI) & $7.9(4.7)$ & $8.6(7.0)$ & $7.6(5.8)$ & $8.0(5.4)$ & 0.916 \\
\hline
\end{tabular}

NT, no treatment; CRS, circadian rhythm support; CBT-I, cognitive behavioral therapy for insomnia; CBT-I+CRS, cognitive behavioral therapy for insomnia combined with circadian rhythm support; ISI, Insomnia Severity Index; PSQI, Pittsburgh Sleep Quality Index; IDS-SR, Inventory of Depressive Symptomatology - Short Form; BAI, Becks

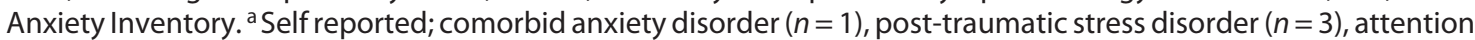
deficit hyperactivity disorder $(n=3)$, autism $(n=1)$, burn-out $(n=3)$, other $(n=1)$. b According to the Composite International Diagnostic Interview - Short Form (CIDI-SF). ${ }^{c}$ Group differences were tested with ANOVA tests for continuous variables, and Fisher Exact tests for categorical variables.

pants that fully completed the interventions) were conducted. Ancillary models evaluated group differences in the change from baseline (T0) to each follow-up (T1-T4) separately. Fisher's exact tests evaluated group differences in the presence of RCI-indicated clinically meaningful worsening or improvement of IDS-SR scores at any of the follow-ups, as well as in CIDI-diagnoses of incident MDD during the 12-month follow-up. Sleep diary variables were evaluated using mixed models with 3 levels (multiple nights nested within assessments at T0, T1, and T4 nested within subjects).

Between-group Cohen's $d$ effect sizes were computed by dividing the group difference in change over time (from baseline to follow-up) by the pooled standard deviations at baseline [48]. Two-sided $p$ values below 0.05 were considered significant. Data were analyzed using R (version 3.6.1) [32].

\section{Results}

\section{Participants}

The RCT included 132 people with ID (73\% female) with a mean age of 48.1 years $(S D=12.8$, range 21-69), experiencing insomnia for 9.7 years on average $(\mathrm{SD}=$ 10.9). At baseline, participants had a mean ISI score of 16.0 ( $\mathrm{SD}=3.8$ ), indicating clinical insomnia of moderate severity [28], and a mean IDS-SR score of $19.3(\mathrm{SD}=7.4)$, indicating mild depressive symptoms [39]. None of the participants had a CIDI diagnosis of MDD at baseline. There were no significant between-group baseline differences in age, sex, insomnia duration, and sleep medica- 
Table 2. Participants characterized in view of the staging model of depression

\begin{tabular}{|c|c|c|c|c|c|c|c|c|c|c|}
\hline & \multicolumn{4}{|c|}{ No current or past MDD } & \multirow{2}{*}{\multicolumn{2}{|c|}{$\frac{\text { No current MDD }}{\text { stage 3: residual phase }}$}} & \multicolumn{4}{|c|}{ Current MDD } \\
\hline & \multicolumn{2}{|c|}{$\frac{\text { stage } 0: \text { no sign }}{\text { IDS-SR } \leq 13}$} & \multicolumn{2}{|c|}{$\frac{\text { stage 1: prodromal }}{\text { IDS-SR }>13}$} & & & \multicolumn{2}{|c|}{$\begin{array}{l}\text { stage } 2 \text { : first episode } \\
\text { new-onset MDD }\end{array}$} & \multicolumn{2}{|c|}{$\begin{array}{l}\text { stage } 4: \text { nth episode } \\
\text { recurrent MDD }\end{array}$} \\
\hline & baseline & 12 months & baseline & 12 months & baseline & 12 months & baseline & 12 months & baseline & 12 months \\
\hline Low-risk NT & $15(50.0 \%)$ & $15(50.0 \%)$ & $2(6.7 \%)$ & $2(6.7 \%)$ & $13(43.3 \%)$ & $11(36.7 \%)$ & $0(0.0 \%)$ & $0(0.0 \%)$ & $0(0.0 \%)$ & $2(6.7 \%)$ \\
\hline High-risk CBT-I & $4(12.9 \%)$ & $3(10.3 \%)$ & $9(29.0 \%)$ & $7(24.1 \%)$ & $18(58.1 \%)$ & $14(48.3 \%)$ & $0(0.0 \%)$ & $2(6.9 \%)$ & $0(0.0 \%)$ & $3(10.3 \%)$ \\
\hline High-risk CBT-I+CRS & $4(12.5 \%)$ & $4(12.9 \%)$ & $11(34.4 \%)$ & $11(35.5 \%)$ & $17(53.1 \%)$ & $14(45.2 \%)$ & $0(0.0 \%)$ & $0(0.0 \%)$ & $0(0.0 \%)$ & $2(6.5 \%)$ \\
\hline
\end{tabular}

Participants can be characterized in view of the staging model of depression according to their CIDI-assessed history of MDD and baseline IDS-SR [49, 50]. The table shows the number and percentage of participants in each stage for each treatment condition both at baseline and at 12-month follow-up. For comparison, staging of the low-risk reference group is also shown. NT, no treatment; CRS, circadian rhythm support; CBT-I, cognitive behavioral therapy for insomnia; CBT-I+CRS, cognitive behavioral therapy for insomnia combined with circadian rhythm support; MDD, major depressive disorder; IDS-SR, Inventory of Depressive Symptomatology - Self-Report.

tion usage, nor were there baseline differences in the severity of symptoms of insomnia or depressive symptoms (all $p>0.867$ ). Table 1 summarizes demographic and clinical characteristics, and Table 2 summarizes the characterization of participants in view of the staging model of depression $[49,50]$. At baseline, $12.9 \%$ could be considered to be in stage 0 (no current or past MDD, IDS-SR $\leq 13$ ); $31.8 \%$ in stage 1 (possible prodromal depression (see Benasi et al. [51] for a critical review) without current or past MDD, IDS-SR $>13$ ); and $55.3 \%$ in stage 3 (residual depression without current MDD, with history of MDD in the past). It should be noted that the discrimination between stage 0 and stage 1 based on an IDS-SR cutoff remains to be validated for use in ID [30].

To validate the expected increased risk in subtype-selected RCT participants, all outcome measures were assessed in parallel in a reference group of 30 people with a subtype of ID without increased risk of depression $(67 \%$ female, age $=52.6, S D=10.9$ ), experiencing insomnia for 10.2 years on average $(\mathrm{SD}=10.3)$. At baseline, ISI was 14.4 $(\mathrm{SD}=3.6)$, IDS-SR was $11.4(\mathrm{SD}=5.6)$, and none of them had a CIDI diagnosis of MDD (see online suppl. material: Supplementary Background for details).

\section{Adherence and Missing Data}

Of the 132 RCT participants, all (100\%) completed the baseline assessments, 130 (98\%) completed the assessments at T1; $125(95 \%)$ at $\mathrm{T} 2 ; 125(95 \%)$ at $\mathrm{T} 3$, and 128 (97\%) at T4 (Fig. 1). Of the 97 participants randomized to one of the active conditions, 89 (91.8\%) completed the intervention fully; 6 (6.2\%) partially (range $1-4$ of the 5 sessions) and remained available for follow-up assessments; and only 2 (2.1\%) dropped out during intervention and were lost to follow-up. None of the 35 participants randomized to NT were lost to follow-up (0\%). As compared to NT, none of the interventions resulted in a significantly higher dropout during the treatment period (Fisher's exact test, all $p>0.232$ ) or subsequent loss to follow-up (Fisher's exact test, all $p>0.115$ ).

\section{Depressive Symptoms Increase without Sleep \\ Intervention}

Table 3 shows group means and intervention effects for depressive symptom severity (IDS-SR). Mixed models evaluated whether depressive symptoms increased without treatment. Confirming the high risk of the included insomnia subtypes, depressive symptom severity during follow-up (T1-T4) increased relative to baseline in the NT group (2.1 [95\% CI 0.1 to 4.0] IDS-SR point increase, $d=$ $0.28, p=0.041)$. In contrast, depressive symptom severity during follow-up (T1-T4) did not significantly differ from baseline in the low-risk reference group $(-0.2[95 \%$ CI -1.7 to 1.4$]$ IDS-SR points, $d=-0.03, p=0.830$ ).

\section{Interventions Prevent Increasing Depressive Symptom Severity}

Relative to the increase in the NT group, depressive symptom severity decreased significantly during T1-T4 after CBT-I ( -5.6 [95\% CI -8.8 to -2.4 ] IDS-SR points, $d$ $=-0.80, p=0.001)$, and after CBT-I+CRS $(-6.3[95 \%$ CI -9.4 to -3.1$], d=-0.95, p<0.001)$, but not after CRS ( $d=$ $-0.24, p=0.199$; see Table 3; online suppl. Table S5; 
Table 3. IDS-SR depression severity and incidence of clinically meaningful worsening or improvement at any of the follow-ups

\begin{tabular}{|c|c|c|c|c|c|c|c|c|}
\hline & \multicolumn{4}{|c|}{ Mean (SD) or $n(\%)$ for each group } & \multicolumn{4}{|c|}{ Intervention effect, $p$ value ${ }^{\mathrm{b}}$} \\
\hline \multicolumn{9}{|l|}{ Depression severity } \\
\hline pre & $19.4(6.2)$ & $19.1(7.9)$ & $19.5(8.2)$ & $18.7(7.5)$ & & & & \\
\hline post (across all 4 follow-ups) & $21.4(7.6)$ & $19.4(9.1)$ & $15.6(9.9)$ & $14.0(7.7)$ & 0.001 & 0.199 & 0.001 & $<0.001$ \\
\hline Clinically meaningful worsening & $15(44 \%)$ & $13(38 \%)$ & $8(28 \%)$ & $3(9 \%)$ & 0.008 & 0.806 & 0.200 & 0.002 \\
\hline Clinically meaningful improvement & $6(18 \%)$ & $9(26 \%)$ & $15(52 \%)$ & $17(53 \%)$ & 0.004 & 0.560 & 0.007 & 0.004 \\
\hline
\end{tabular}

Significance of intervention effects overall and for between-group contrasts. NT, no treatment; CRS, circadian rhythm support; CBT-I, cognitive behavioral therapy for insomnia; CBT-I+CRS, cognitive behavioral therapy for insomnia combined with circadian rhythm support. ${ }^{a}$ Clinically meaningful change at any of the follow-ups based on the Reliable Change Index (RCI) using the baseline standard deviation and a test-retest reliability of 0.87 , including participants completing $\geq 1$ follow-up. ${ }^{\mathrm{b}}$ Tested with linear-mixed models for depression severity and Fisher Exact tests for clinical change.

Fig. 2). The decrease observed in the CBT-I and CBTI+CRS groups did not differ $(d=-0.10, p=0.679$; see online suppl. Table S6).

The online supplementary material shows ancillary analyses on individual follow-up timepoints, adapted IDS-SR scores excluding sleep items, models including covariates, and per-protocol analyses (online suppl. Table S5-S7).

\section{Clinically Meaningful Worsening and Improvement}

Figure 3 shows the percentage of participants experiencing an RCI-based clinically meaningful worsening or improvement of depressive symptoms in each treatment condition at every follow-up, suggesting the best all-year prevention by CBT-I+CRS. Indeed, clinically meaningful worsening of depressive symptoms occurred at least once during the 1-year follow-up in $44 \%$ of the participants in the NT condition, versus $38 \%, 28 \%$, and $9 \%$ of the participants in, respectively, the CRS, CBT-I, and CBTI+CRS conditions (Fisher's exact test, $p=0.008$ ). Between-group comparisons (Table 3 ) showed that only CBT-I+CRS differed from NT $(p=0.002)$, as well as from CRS $(p=0.009)$ - an active control condition.

A clinically meaningful improvement of depressive symptoms occurred at least once during the 1-year follow-up in $18 \%$ of the participants in the NT condition, versus $26 \%, 52 \%$, and $53 \%$ of the participants in, respectively, the CRS, CBT-I, and CBT-I+CRS conditions ( $p=$ 0.004 ). Between-group comparisons (Table 3) showed that CBT-I+CRS differed from NT ( $p=0.004)$, as well as from CRS $(p=0.043)$ - an active control condition. CBT-

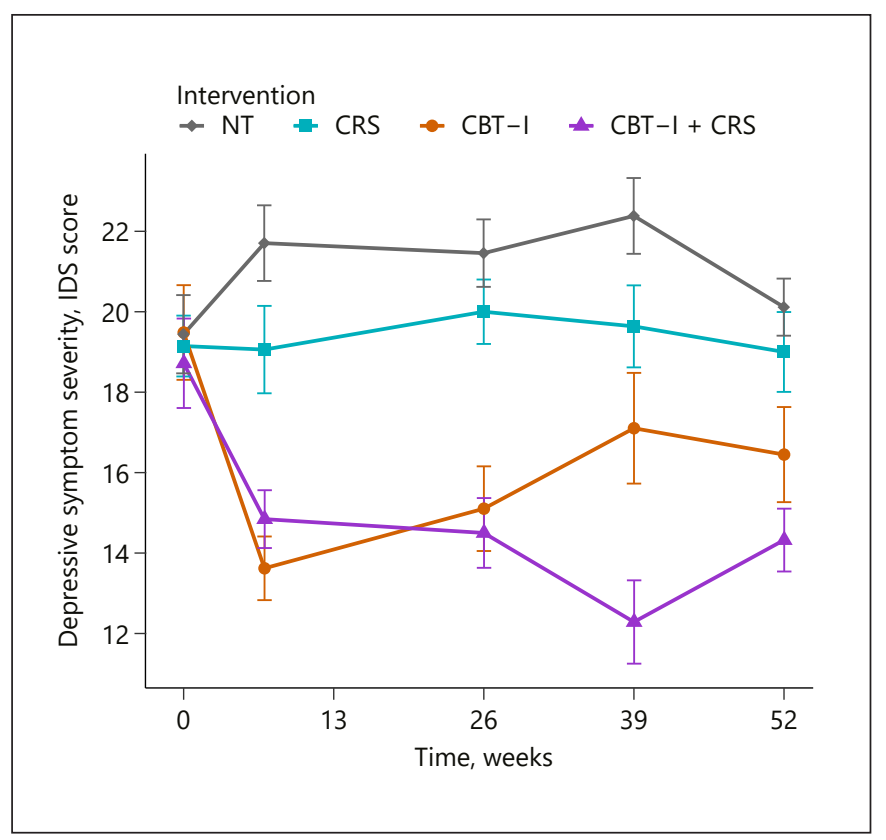

Fig. 2. Mean depressive symptom severity scores for each treatment condition at T0, T1, T2, T3, and T4. Error bars represent the standard error. NT, no treatment; CRS, circadian rhythm support; CBT-I, cognitive behavioral therapy for insomnia; CBT-I+CRS, combined cognitive behavioral therapy for insomnia with circadian rhythm support.

I also differed from NT ( $p=0.007)$, but not from CRS $(p=0.067)$.

Attesting to the importance of repeated assessments, the CIDI assessed at T4 as a secondary outcome was not 


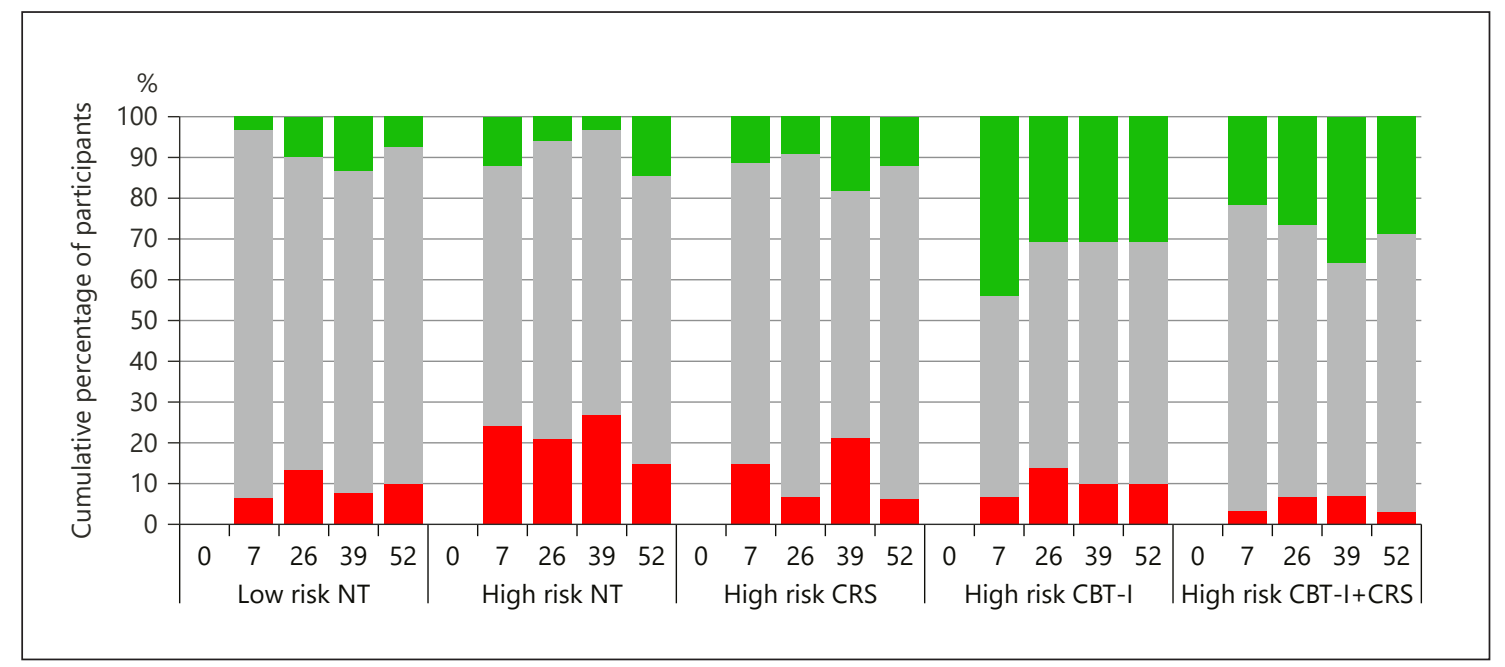

Fig. 3. RCI-based clinically meaningful worsening and improvement of depressive symptom severity at individual follow-ups. The cumulative percentages of participants with reliable worsening (red), improvement (green), or no reliable change (grey) of depression severity at each follow-up (week 7, 26, 39, and 52) for a low-risk insomnia reference control group (leftmost bars) and all conditions for high-risk insomnia. NT, no treatment; CRS, circadian rhythm support; CBT-I, cognitive behavioral therapy for insomnia; CBT-I+CRS, combined cognitive behavioral therapy for insomnia with circadian rhythm support.

sufficiently sensitive [52] to retrospectively detect these episodes of clinically meaningful increased severity of depressive symptoms that the RCI identified to have occurred over the year. The CIDI detected MDD in 4/35 (11.4\%) of the participants in the NT condition, versus $4 / 33$ (12.1\%), 5/29 (17.2\%), and 2/31 (6.5\%) in the CRS, CBT-I, and CBT-I+CRS conditions, respectively. The numbers were too small to lead to statistically significant group differences (Fisher's exact test, $p=0.650$ ).

\section{Secondary Sleep Outcomes}

Insomnia Severity

Compared to the NT group, insomnia symptoms became significantly less severe from baseline to T1-T4 after CBT-I ( -3.9 [95\% CI -6.0 to -1.9 ] ISI-points, $d=-0.98$, $p<0.001)$ and CBTI-I+CRS $(-3.0$ [95\% CI -5.0 to -1.0$]$ ISI-points, $d=-0.79, p=0.003$ ) but not after CRS (see online suppl. Table S5). The supplement shows ancillary analyses on individual follow-up timepoints (see online suppl. material: Supplementary Results; Table S5; Fig. S1).

\section{Sleep Diary Variables}

Compared to the NT group, sleep efficiency increased from baseline to T1-T4 after CBT-I ( $5.5 \%$ [ $95 \%$ CI 0.8 to $10.2], d=0.42, p=0.023)$ and CBT-I+CRS $(6.7 \%$ [95\% CI
2.0 to 11.4$], d=0.50, p=0.005$; see online suppl. Table S5). Likewise, compared to the NT group, WASO decreased from baseline to T1-T4 after CBT-I (-26.6 min [95\% CI -49.2 to -4.0$], d=-0.46, p=0.021)$ and CBTI+CRS $(-31.4 \min$ [95\% CI -53.8 to -8.9 ] $, d=-0.53, p=$ 0.006). CRS did not improve sleep efficiency or WASO. None of the interventions altered sleep onset latency or total sleep time. The supplement shows ancillary analyses on individual follow-up timepoints (see online suppl. material: Supplementary Results; Table S5; Fig. S2-S5).

\section{Side Effects}

None of the participants reported adversities. Furthermore, active inquiry about complaints indicated no timeby-treatment effects (online suppl. Table S4).

\section{Discussion}

The current study addressed two crucial requirements to facilitate the feasibility and efficiency of preventing worsening of depressive symptoms [3-5]. First, preventive strategies would most efficiently be evaluated and implemented if the target group can be selected for having an increased risk [6]. Second, the strategy should have significant and clinically meaningful preventive effects in 
the target group. Our RCT in people with insomnia selected for an increased risk of depression indicate that therapist-guided digital CBT-I, especially in combination with CRS, prevents the worsening of depressive symptoms during the year to follow.

Previous studies on the possibility to prevent depression by treating the major risk factor of insomnia were inconclusive. First, depressive symptoms decreased as well in the control groups [11-13]. These studies thus included people with insomnia that were, on average, heading towards spontaneous improvement of depressive symptoms anyway. The studies demonstrated CBT-I to enhance pending relief of depressive symptoms rather than prevention of their worsening. Second, the studies reported a very high dropout rate, possibly biased to have occurred especially among the more depressed participants. Our study successfully solved these issues by selecting insomnia subtypes with the highest risk of MDD [7] and by preventing dropout through therapist guidance.

By repeatedly assessing depressive symptoms at four follow-ups spanning a year, we indeed showed that untreated high-risk insomniacs experienced significant and clinically meaningful worsening of depressive symptoms. In an untreated low-risk reference group, depressive symptom severity during follow-up did not differ significantly from baseline. The demonstrated possibility to select participants at increased risk fulfills a first requirement for efficient evaluation and implementation of strategies aiming for the prevention of depression.

The next requirement is to demonstrate that this target group responds favorably to an intervention that ideally is scalable. In contrast to previous fully automated digital approaches [11-13], the current study is the first to demonstrate that therapist-guided CBT-I, especially in combination with CRS, can specifically prevent worsening of depressive symptoms during a 1-year follow-up in high-risk insomniacs (Fig. 2, 3). The post-treatment (at T1) effect of guided digital CBTI on depressive symptom severity (Cohen's $d=-1.11$ ) compares favorably to meta-analyzed effects of previous digital CBT interventions addressing either insomnia symptoms (Hedges' $g=-0.35$ [53]) or depressive symptoms $(d=-0.57$ [54]; see online suppl. material: Supplementary Discussion for details). The demonstrated effectiveness in the target group of high-risk insomniacs fulfills the second requirement for efficient evaluation and implementation of strategies aiming to prevent worsening of depressive symptoms.

Our study evaluated whether effects of CBT-I on depressive symptoms can be enhanced by adding CRS. A previous study found no sleep-improving effect of standalone CRS [20]. Adding CRS to CBT-I moreover did not boost the effects of CBT-I immediately after treatment. However, effects did emerge at later follow-up, indicating that the addition of CRS resulted in a better maintenance of beneficial effects initially achieved by CBT-I. Also in the current study, stand-alone CRS did not significantly improve either sleep or depressive symptoms, in line with the only meta-analysis on one of the CRS interventions (physical activity) specifically in insomnia patients [19]. However, as observed before [20], also in our study the addition of CRS to CBT-I appeared to better maintain or even boost late effects of CBT-I on sleep diary variables (online suppl. Fig. S2-S5), as well as on depressive symptoms (Fig. 1). In fact, only CBT-I combined with CRS reduced the 1-year incidence of clinically meaningful worsening of depression. The usefulness of combined or sequential treatment was proposed already more than 20 years ago [55]. Relevant for insomnia and depression, $\mathrm{Pi}$ geon et al. [56] recently demonstrated in people with posttraumatic stress disorder (PTSD) that the sequential delivery of CBT-I and routine cognitive processing therapy (CPT) improved insomnia, depression, and PTSD symptoms significantly better than stand-alone CPT did.

In addition to validating the vulnerability of a targetable group, and optimizing the long-term intervention effectiveness in this group, other strengths of our study are the adherence to interventions and the limited dropout and loss to follow-up. Previous studies using fully automated digital CBT-I were difficult to interpret because of high dropout [11-13], which may have occurred especially in patients with more severe depressive symptoms $[14,15]$. Therapy guidance enhances adherence by additional explanation, motivation, and support [57]. Next to the high adherence and limited dropout from intervention, our loss to follow-up assessments was also very low. Factors other than therapist guidance during the intervention must be involved, because loss to follow-up was equally low in the NT group, who never received therapist guidance. A probable reason is that this RCT was part of a larger multimodal investigation including, for example, sleep EEG, MRI, and ambulatory monitoring [23]. Consequently, participants of all four groups received a considerable and equal amount of attention from researchers.

Limitations of the present study need to be considered. First, although we observed half as many CIDI diagnoses of MDD in the CBT-I+CRS group compared to the NT, CBT-I, and CRS groups ( $n=2$ versus 4,5 , and 4 ; $6.5 \%$ versus $11.4 \%, 17.2 \%$, and $12.1 \%$, respectively), the 
numbers were too small for statistical tests to demonstrate significant group differences. In hindsight, the CIDI may not have been the best choice for a secondary outcome measure to assess a clinical diagnosis of MDD or to assess changes over time. A recent meta-analysis showed that the CIDI has less sensitivity to changes in symptom severity and classifies MDD only half as often as the MINI structured interview [52]. Insensitivity may have precluded sufficient statistical power to detect the group-by-time interaction effects that were so evident in the primary outcome measure - whether analyzed with linear mixed effect models or with the RCI. A related limitation is the duration of the follow-up period, which spanned 1 year only. Future studies could increase sensitivity by extending the follow-up period to multiple years. A limitation with respect to the interpretation of our findings on adherence and dropout is that this RCT was part of a larger multimodal investigation [23]. This embedding may have contributed to the adherence of the participants.

Our findings are particularly relevant in light of the notion that prevention is essential to combat the global burden and cost of MDD [3-5]. Therapist-guided digital CBT-I, especially in combination with CRS, for people with insomnia selected for a particularly high risk of developing depression, appears to be a promising and feasible approach. CBT-I+CRS may be considered by healthcare institutes and government prevention programs as an effective scalable intervention to prevent the worsening of depressive symptoms.

\section{Acknowledgements}

We would like to thank Bart te Lindert, Annemieke van Straten, and Rick Wassing for their contribution to the development and implementation of the digital treatment modules. We would also like to thank Sietkse Bootsma, Tom Bresser, Jennifer Ramautar, Seline Roelvink, Lara Rösler, Chin Tong, and Laura Vergeer for their help with the data collection and preprocessing.

\section{Statement of Ethics}

This study is in accordance with the ethical standard of relevant national and international committees on human experimentation and with the declaration of Helsinki. All participants provided written informed consent, and the study protocol was approved by the Medical Ethics Committee of the VU University Medical Centre (NL63139.029.17) and registered with the Netherlands Trial Register (NL7359).

\section{Conflict of Interest Statement}

J.La. and T.v.d.Z. developed the digital CBT-I treatment module supported by Governmental Support through research grants, and without any commercial interest. None of the authors have a conflict of interest to declare.

\section{Funding Sources}

This work received funding from the European Research Council ERC-ADG-2014-671084. O.L.-K., J.Le., J.R., and T.B. were supported by Vrije Universiteit Amsterdam University Research Fellowships.

\section{Author Contributions}

E.V.S. is the principal investigator of this trial. J.Le. and J.F.-D. coordinated the trial, contributed to the design and implementation of the trial, and collected the data. J.La. and T.v.d.Z. developed the CBT-I intervention, T.M. developed the CRS module. T.B. randomized the participants and contributed to the design of the trial. L.D. coordinated the therapy guidance, trained and supervised the other therapists, and collected data. O.L.-K., A.A., S.I., and S.M. recruited participants, provided therapy guidance, and collected data. J.R., G.v.d.L. and S.v.d.V. collected data. J.Le. performed the statistical analyses. J.Le. and E.V.S. interpreted the results and drafted the manuscript. All authors reviewed and approved the manuscript.

\section{Data Availability Statement}

The data that support the findings of this study are available from the corresponding author upon reasonable request.

\section{References}

Psychother Psychosom 2022;91:168-179

DOI: $10.1159 / 000520282$
1 World Health Organization. Depression and other common mental disorders: global health estimates. Geneva: World Health Organization; 2017.

2 Verduijn J, Verhoeven JE, Milaneschi Y, Schoevers RA, van Hemert AM, Beekman ATF, et al. Reconsidering the prognosis of major depressive disorder across diagnostic boundaries: full recovery is the exception rather than the rule. BMC Med. 2017 Dec 12; 15(1):215.

3 Cuijpers P, Beekman AT, Reynolds CF 3rd. Preventing depression: a global priority. JAMA. 2012 Mar 14;307(10):1033-4.

4 Munoz RF, Bunge EL. Prevention of depression worldwide: a wake-up call. Lancet Psychiatry. 2016 Apr;3(4):306-7.

5 Ebert DD, Cuijpers P. It is time to invest in the prevention of depression. JAMA Netw Open. 2018;1(2):e180335.

Treating Insomnia to Prevent Worsening 
6 Munoz RF, Cuijpers P, Smit F, Barrera AZ, Leykin Y. Prevention of major depression. Annu Rev Clin Psychol. 2010;6:181-212.

7 Blanken TF, Benjamins JS, Borsboom D, Vermunt JK, Paquola C, Ramautar J, et al. Insomnia disorder subtypes derived from life history and traits of affect and personality. Lancet Psychiatry. 2019 Feb;6(2):151-63.

8 Riemann D, Baglioni C, Bassetti C, Bjorvatn B, Dolenc Groselj L, Ellis JG, et al. European guideline for the diagnosis and treatment of insomnia. J Sleep Res. 2017;26(6):675-700.

9 Soh HL, Ho RC, Ho CS, Tam WW. Efficacy of digital cognitive behavioural therapy for insomnia: a meta-analysis of randomised controlled trials. Sleep Med. 2020 Nov;75:315-25.

10 Ho FY, Chan CS, Lo WY, Leung JC. The effect of self-help cognitive behavioral therapy for insomnia on depressive symptoms: an updated meta-analysis of randomized controlled trials. J Affect Disord. 2020 Mar 15;265:287304.

11 Christensen H, Batterham PJ, Gosling JA, Ritterband LM, Griffiths KM, Thorndike FP, et al. Effectiveness of an online insomnia program (SHUTi) for prevention of depressive episodes (the GoodNight Study): a randomised controlled trial. Lancet Psychiatry. 2016;3(4):333-41.

12 Batterham PJ, Christensen H, Mackinnon AJ, Gosling JA, Thorndike FP, Ritterband LM, et al. Trajectories of change and long-term outcomes in a randomised controlled trial of internet-based insomnia treatment to prevent depression. BJPsych Open. 2017;3(5):228-35.

13 Cheng P, Kalmbach DA, Tallent G, Joseph CL, Espie CA, Drake CL. Depression prevention via digital cognitive behavioral therapy for insomnia: a randomized controlled trial. Sleep. 2019 Oct 9;42(10):zsz150.

14 Asarnow LD, Manber R. Cognitive behavioral therapy for insomnia in depression. Sleep Med Clin. 2019;14(2):177-84.

15 Ong JC, Kuo TF, Manber R. Who is at risk for dropout from group cognitive-behavior therapy for insomnia? J Psychosom Res. 2008 Apr; 64(4):419-25.

16 Wirz-Justice A, Terman M, Oren DA, Goodwin FK, Kripke DF, Whybrow PC, et al. Brightening depression. Science. 2004 Jan 23; 303(5657):467-9.

17 Wirz-Justice A. Chronobiology and psychiatry. Sleep Med Rev. 2007 Dec 1;11(6):423-7.

18 Van Someren EJW, Riemersma RF, Swaab DF. Functional plasticity of the circadian timing system in old age: light exposure. Prog Brain Res. 2002;138:205-31.

19 Banno M, Harada Y, Taniguchi M, Tobita R, Tsujimoto H, Tsujimoto Y, et al. Exercise can improve sleep quality: a systematic review and meta-analysis. PeerJ. 2018 Jul 11;6:e5172.

20 Dekker K, Benjamins JS, Maksimovic T, Filardi M, Hofman WF, van Straten A, et al. Combined internet-based cognitive-behavioral and chronobiological intervention for insomnia: a randomized controlled trial. Psychother Psychosom. 2020;89(2):117-8.
21 Al-Karawi D, Jubair L. Bright light therapy for nonseasonal depression: meta-analysis of clinical trials. J Affect Disord. 2016 Jul 1;198: 64-71.

22 Stubbs B, Vancampfort D, Hallgren M, Firth J, Veronese N, Solmi M, et al. EPA guidance on physical activity as a treatment for severe mental illness: a meta-review of the evidence and Position Statement from the European Psychiatric Association (EPA), supported by the International Organization of Physical Therapists in Mental Health (IOPTMH). Eur Psychiatry. 2018;54:124-44

23 Leerssen J, Foster-Dingley JC, Lakbila-Kamal O, Dekkers LMS, Albers ACW, Ikelaar SLC, et al. Internet-guided cognitive, behavioral and chronobiological interventions in depression-prone insomnia subtypes: protocol of a randomized controlled prevention trial. BMC Psychiatry. 2020 Apr 15;20(1):163.

24 Guidi J, Brakemeier EL, Bockting CLH, Cosci F, Cuijpers P, Jarrett RB, et al. Methodological recommendations for trials of psychological interventions. Psychother Psychosom. 2018; 87(5):276-84

25 American Psychiatric Association. Diagnostic and statistical manual of mental disorders. 5th ed. Washington: American Psychiatric Press; 2013.

26 Sateia MJ. International classification of sleep disorders-third edition: highlights and modifications. Chest. 2014;146(5):1387-94.

27 Bastien CH, Vallières A, Morin CM. Validation of the Insomnia Severity Index as an outcome measure for insomnia research. Sleep Med. 2001 Jul;2(4):297-307.

28 Morin CM, Belleville G, Bélanger L, Ivers H. The Insomnia Severity Index: psychometric indicators to detect insomnia cases and evaluate treatment response. Sleep. 2011;34(5): 601-8.

29 Tunis SR, Stryer DB, Clancy CM. Practical clinical trials: increasing the value of clinical research for decision making in clinical and health policy. JAMA. 2003 Sep 24;290(12): 1624-32.

30 Carney CE, Ulmer C, Edinger JD, Krystal AD, Knauss F. Assessing depression symptoms in those with insomnia: an examination of the Beck Depression Inventory second edition (BDI-II). J Psychiatr Res. 2009 Feb;43(5):57682.

31 Kang M, Ragan BG, Park JH. Issues in outcomes research: an overview of randomization techniques for clinical trials. J Athl Train. 2008;43(2):215-21.

32 R Core Team. R: a language and environment for statistical computing. Vienna: R Foundation for Statistical Computing; 2019.

33 van Straten A, Emmelkamp J, de Wit J, Lancee J, Andersson G, van Someren EJ, et al. Guided Internet-delivered cognitive behavioural treatment for insomnia: a randomized trial. Psychol Med. 2014 May;44(7):1521-32.
34 van der Zweerde T, van Straten A, Effting M, Kyle SD, Lancee J. Does online insomnia treatment reduce depressive symptoms? A randomized controlled trial in individuals with both insomnia and depressive symptoms. Psychol Med. 2019 Feb;49(3):501-9.

35 Van der Zweerde T, Lancee J, Slottje P, Bosmans JE, Van Someren EJW, van Straten A. Nurse-guided internet-delivered cognitive behavioral therapy for insomnia in general practice: results from a pragmatic randomized clinical trial. Psychother Psychosom. 2020;89(3):174-84

36 Fava GA, Rafanelli C, Tomba E. The clinical process in psychiatry: a clinimetric approach. J Clin Psychiatry. 2012;73(2):177-84.

37 Hunsley J, Meyer GJ. The incremental validity of psychological testing and assessment: conceptual, methodological, and statistical issues. Psychol Assess. 2003;15(4):446-55.

38 Carrozzino D, Patierno C, Guidi J, Berrocal Montiel C, Cao J, Charlson ME, et al. Clinimetric criteria for patient-reported outcome measures. Psychother Psychosom. 2021; 90(4):222-32.

39 Rush AJ, Gullion CM, Basco MR, Jarrett RB, Trivedi MH. The Inventory of Depressive Symptomatology (IDS): psychometric properties. Psychol Med. 1996 May;26(3):477-86.

40 Schulte-van Maaren YW, Carlier IV, Zitman FG, van Hemert AM, de Waal MW, van der Does AJ, et al. Reference values for major depression questionnaires: the Leiden Routine Outcome Monitoring Study. J Affect Disord. 2013 May 1;149(1):342-9.

41 Jacobson NS, Truax P. Clinical significance: a statistical approach to defining meaningful change in psychotherapy research. J Consult Clin Psychol. 1991;59(1):12-9.

42 Aslan S, Kuruoglu AC, Isikli S, Rush J, Taner E, Isik E. The Inventory of Depressive Symptomatology (IDS): validity and reliability of clinician (IDS-C30) and self-report form (IDS-SR30) in patients with major depressive disorder. Bull Clin Psychopharmacol. 2006; 16(1):1-14.

43 Kessler RC, Andrews G, Mroczek D, Ustun B, Wittchen H-U. The World Health Organization Composite International Diagnostic Interview short-form (CIDI-SF). Int J Method Psychiat Res. 1998;7(4):171-85.

44 Carney CE, Buysse DJ, Ancoli-Israel S, Edinger JD, Krystal AD, Lichstein KL, et al. The consensus sleep diary: standardizing prospective sleep self-monitoring. Sleep. 2012 Feb 1; 35(2):287-302.

45 Reed DL, Sacco WP. Measuring sleep efficiency: what should the denominator be? J Clin Sleep Med. 2016 Feb;12(2):263-6.

46 Hakkaart-van Roijen L, Straten A, Tiemens B, Donker CHM. Trimbos/iMTA questionnaire for costs associated with psychiatric illness (TIC-P). Inst Med Technol Assess. 2002. Dutch.

47 Twisk JWR. Applied longitudinal data analysis for epidemiology. Cambridge: Cambridge University Press; 2003. 
48 Morris SB. Estimating effect sizes from pretest-posttest-control group designs. Organ Res Methods. 2008 April 1;11(2):364-86.

49 Cosci F, Fava GA. Staging of mental disorders: systematic review. Psychother Psychosom. 2013;82(1):20-34.

50 Verduijn J, Milaneschi Y, van Hemert AM, Schoevers RA, Hickie IB, Penninx BW, et al. Clinical staging of major depressive disorder: an empirical exploration. J Clin Psychiatry. 2015 Sep;76(9):1200-8.

51 Benasi G, Fava GA, Guidi J. Prodromal symptoms in depression: a systematic review. Psychother Psychosom. 2021;90(6):365-72.
$52 \mathrm{Wu}$ Y, Levis B, Sun Y, Krishnan A, He C, Riehm KE, et al. Probability of major depression diagnostic classification based on the SCID, CIDI and MINI diagnostic interviews controlling for Hospital Anxiety and Depression Scale - Depression subscale scores: an individual participant data meta-analysis of 73 primary studies. J Psychosom Res. 2020 Feb;129:109892.

53 Ho FY, Chan CS, Lo WY, Leung JC. The effect of self-help cognitive behavioral therapy for insomnia on depressive symptoms: an updated meta-analysis of randomized controlled trials. J Affect Disord. 2020;265:287-304.

54 Karyotaki E, Efthimiou O, Miguel C, Bermpohl FM, Furukawa TA, Cuijpers P, et al. Internet-based cognitive behavioral therapy for depression: a systematic review and individual patient data network meta-analysis. JAMA Psychiatry. 2021;78(4):361-71.
55 Fava GA. Sequential treatment: a new way of integrating pharmacotherapy and psychotherapy. Psychother Psychosom. 1999;68(5): 227-9.

56 Pigeon WR, Crean HF, Cerulli C, Gallegos AM, Bishop TM, Heffner KL. A randomized clinical trial of cognitive-behavioral therapy for insomnia to augment posttraumatic stress disorder treatment in survivors of interpersonal violence. Psychother Psychosom. 2021.

57 Lancee J, van den Bout J, Sorbi MJ, van Straten A. Motivational support provided via email improves the effectiveness of internet-delivered self-help treatment for insomnia: a randomized trial. Behav Res Ther. 2013 Dec 1; 51(12):797-805. 